\title{
FERNANDO REIMERS*
}

\section{APRENDER A LEER Y A CONVIVIR EN LAS ESCUELAS RURALES EN COLOMBIA ${ }^{1}$}

\begin{abstract}
Resumen
El texto que aquí se presenta es un análisis de la situación de las Escuelas Nuevas en Colombia. Se abordan los contextos que como obstáculos han entrabado el desarrollo de la educación en la población infantil y se plantean algunas salidas. Estructuralmente el texto describe tres desafíos para los maestros de este tipo de escuelas. El primero se refiere a los alumnos que no ingresan a la escuela o que desertan antes de culminar la educación básica. El segundo desafío tiene que ver con las débiles competencias de lectoescritura y las débiles culturas escolares de los niños de las escuelas rurales. El tercer desafío hace referencia a la insuficiente atención al desarrollo de competencias para la convivencia. La exposición de cada uno de estos desafíos se apoya en una serie de documentos con abundante y detallada información. Finalmente, el documento presenta algunas sugerencias para atender estos desafíos.
\end{abstract}

Palabras clave: Escuelas nuevas, educación básica, deserción, profesores, niños.

\begin{abstract}
This text is an analysis of the situation of the New Schools in Colombia. The contexts are approached to obstacles they have hindered the development of the education in the infantile population and they think about some exits. Structurally the text describes three challenges for the teachers of this kind of schools. The first one refers to the students that don't enter to the school or that they desert before culminating the basic education. The second challenge has to deal with the weak reading - writing competitions and the weak school cultures of the children of the rural schools. The third challenge makes reference to the insufficient attention to the development of competitions for the coexistence. The exhibition of each one of these challenges leans on in a series of documents with abundant and detailed information. Finally, the document presents some suggestions to assist these challenges.
\end{abstract}

Key words: New schools, basic education, desertion, teacher, childs.

* Universidad de Harvard - reimerfe@gse.harvard.edu

1 Este trabajo fue presentado el día 4 de marzo de 2003 en el marco del Congreso Internacional de Escuelas Nuevas, llevado a cabo en la ciudad de Armenia, organizado por la Fundación Volvamos a la Gente. 
Aprecio mucho la oportunidad de estar con ustedes en este Congreso de Escuelas Nuevas, por varias razones. En primer lugar, porque asumo que muchos de ustedes tienen un interés especial en mejorar la calidad de las Escuelas Rurales, es decir, de las escuelas donde los estudiantes tienen peores oportunidades de aprender en América Latina. En segundo lugar, porque la historia de Escuela Nueva es fascinante para todo el que se interese en estudiar el proceso del cambio educativo. La historia de cómo Oscar Mogollón y un grupo de maestros se reunieron para inventar desde la escuela rural un enfoque que fuese más apropiado a sus circunstancias, es inspiradora en tanto nos recuerda el enorme potencial que existe en las escuelas y en la iniciativa de cada maestro. La historia de cómo Vicky Colbert, desde el Ministerio de Educación, apoyó esta iniciativa y su expansión, es un ejemplo maravilloso de cómo el Gobierno central puede jugar un excelente papel cuando apoya la iniciativa local. La historia de la creación de la Fundación Volvamos a la Gente, es un buen ejemplo de cómo la sociedad civil organizada tiene un papel fundamental que jugar en la creación de conciencia pública sobre la calidad de la educación y en hacer que el Estado ejerza con responsabilidad y transparencia la confianza que en los funcionarios públicos han delegado los ciudadanos al encomendarles la administración de los recursos que financian quienes pagan impuestos para lograr fines públicos. Finalmente, la historia de cómo diversas organizaciones nacionales e internacionales han apoyado el proyecto Escuela Nueva a lo largo del tiempo, demuestra que es posible la cooperación efectiva sobre proyectos que tienen sentido.

Recuerdo aún mi primera visita a una Escuela Nueva en Zipaquirá hace una década. Nos recibió el presidente de la escuela, Juan Gómez, quien tenía entonces unos 10 años y quien procedió a explicarnos el Gobierno Escolar, a mostrarnos el mapa de su localidad, a explicarnos cómo funcionaba la escuela y a mostrarnos sus cuadernos de trabajo y lo que había aprendido en su cuarto grado. Venía yo de pasar tres meses visitando escuelas rurales en otro país de América Latina y la visita a Juan Gómez y sus compañeros me sirvió para sacudirme la tristeza que me había producido ver escuelas en condiciones muy deficientes. Conozco de varios estudios que hablan de los efectos positivos de Escuela Nueva, de su exitoso proceso de implementación y consolidación.

Sin embargo, lo que más admiro de Escuela Nueva ha sido su capacidad de renovarse permanentemente, fundamental en una innovación que tiene ya varias décadas de vida, así como su capacidad de estimular el dialogo y la discusión crítica sobre los propósitos y las formas más efectivas de educar a los niños en zonas rurales, que es decir a los más pobres en América Latina. Este Congreso es un ejemplo de esa capacidad de Escuela Nueva de facilitar el dialogo crítico. Si Escuela Nueva no hubiese cambiado a lo largo de su existencia, o si los esfuerzos por extenderla se limitasen a tratar de 'embotellar' un par de recetas que caracterizan a las Escuelas Nuevas para distribuirlas en distintos contextos, no sería yo tan entusiasta de los esfuerzos de quienes han sido responsables por el éxito de esta innovación educativa.

La razón por la que creo que es especialmente importante valorar una organización como Escuela Nueva, que permite el diálogo crítico sobre el propósito y los medios de educar a los más pobres en América Latina, aquellos que viven en zonas rurales, es porque creo que las oportunidades de que los hijos de los pobres aprendan resultan siempre de un equilibrio precario entre quienes piensan que la educación en América Latina debe servir a un proyecto incluyente de sociedad y quienes piensan, por el contrario, que la educación debe servir para reproducir y consolidar un orden social que refleja demasiado el origen feudal de las sociedades latinoamericanas ${ }^{2}$. Creo que en ese equilibrio precario por crear oportunidades efectivas de aprendizaje significativo para los hijos de los pobres, hay tanto avances como retrocesos, y los retrocesos toman a veces mucho menos tiempo que el necesario para consolidar avances. Creo que la historia misma de Escuela Nueva, en distintas épocas en Colombia, ilustra en parte, estas luchas y estos avances y retrocesos.

Quisiera, en lo que resta de este trabajo y sabiendo que les anima a ustedes el deseo de profundizar en la conquista de nuevos desafíos educativos, en el ánimo de seguir reinventando a Escuela Nueva y otros programas de Educación Rural en América Latina para hacerlos más pertinentes y más efectivos, mencionar tres desafíos de especial importancia para los maestros en escuelas rurales en Colombia: los alumnos que no ingresan a la escuela y que desertan antes de culminar la educación básica, las débiles competencias de lectoescritura y las débiles culturas escolares, y la insuficiente atención al desarrollo de competencias para la convivencia. Finalmente, desarrollaré algunas sugerencias para atender a estos desafíos.

\section{Primer desafió. Todavía hay estudiantes que no terminan la educación primaria. La deserción está aumentando}

Durante los últimos cincuenta años Colombia logro expandir notablemente el acceso inicial a la educación primaria (a pesar de que ya la Constitución de 1886 establecía el derecho a la educación primaria universal y gratuita). Como resultado la gran mayoría de los niños inician actualmente el primer grado, con diferencias relativamente menores en la oportunidad de acceso ini-

\footnotetext{
2 Ver Reimers, Fernando. "La lucha por la igualdad de oportunidades educativas en América Latina como proceso político". Revista Latinoamericana de Estudios Educativos. Vol. 32. Número 1. 2002, págs. 9-70.
} 
cial de distintos grupos socioeconómicos. Sin embargo, sólo una pequeña proporción de los niños que inician la escuela primaria la terminan; la mayoría de quienes desertan son los niños de las familias más pobres y la deserción es muy superior en zonas rurales. Esto resulta en dos consecuencias graves para el funcionamiento de una democracia y para la paz. Por una parte, los niveles educativos de la población son bajos y, por otra parte, hay brechas educativas significativas entre distintos grupos sociales que sugieren que las oportunidades educativas reflejan las diferencias sociales de origen entre las personas.

En Colombia el nivel educativo de la población económicamente activa es muy inferior a los niveles educativos de la población en países de la OECD. El $39 \%$ de la población en Colombia ha alcanzado apenas alguna educación primaria, el $7 \%$ no tiene educación, el $21 \%$ ha cursado alguna educación secundaria y sólo han completado la secundaria el $20 \%$. El $5.6 \%$ han cursado, pero no concluido, estudios universitarios y el $8 \%$ han concluido estudios universitarios ${ }^{3}$. Esto compara con promedios en la OECD, donde $16 \%$ tiene solo educación primaria y un $20 \%$ adicional solo educación secundaria. En otras palabras, en promedio, 2.3 de cada 5 personas, en Colombia tienen apenas educación primaria o menos, comparado con 0.8 de cada 5 personas que tienen ese nivel en los países de la OECD. Por otra parte, 3 de cada 5 personas en los países de la OECD han completado al menos el nivel superior de educación secundaria en comparación con Colombia, donde apenas 1.7 de cada 5 personas ha completado este nivel. El contraste es claro, un niño nacido en Colombia tiene una probabilidad de terminar apenas la primaria, que es 3 veces la que tiene un niño nacido en la OECD, mientras que

\footnotetext{
${ }^{3}$ Sarmiento-Gómez, Alfredo. 2002. "Política y Equidad Educativas en Colombia". En Reimers, F. (Ed). Distintas escuelas, diferentes oportunidades. Los retos para la igualdad de oportunidades en Latinoamérica. Madrid: Editorial Arco, página 347.
}

un niño nacido en un país de la OECD tiene una probabilidad de completar la secundaria, que es 1.8 veces la de uno nacido en Colombia.

La baja escolaridad de las personas debilita las capacidades de ejercer ciudadanía. En un estudio sobre los factores que inciden en las actitudes políticas de las personas llevado a cabo en 48 países, entre 1995-1997, se encuentran significativas diferencias en las actitudes democráticas de las personas con distintos niveles educativos, siendo las más democráticas aquellas con mayores niveles escolaridad $^{4}$. Igualmente, las personas con más escolaridad son aquellas que confían más en las demás personas. La confianza interpersonal es un importante predictor de la orientación democrática de las personas.

El problema de la baja escolaridad en Colombia se agrava porque al concentrarse la menor oportunidad educativa entre los niños de las familias más pobres, esto reproduce el círculo vicioso de la pobreza.

Quienes no acceden a completar la educación básica, y por lo tanto a los niveles superiores de educación, son los más pobres. En Colombia, la mayoría de los niños comienzan el primer grado en algún momento en su vida. En el año 2000 , el $99 \%$ de los niños de 12 años, en las familias con el $20 \%$ con más ingresos habían estado matriculados alguna vez en la escuela, y entre el $40 \%$ de las familias con menos ingresos, el $96 \%$ de

\footnotetext{
${ }^{4}$ Ai Camp, Roderic. 2001. Citizen Views of Democracy in Latin America. Pittsburgh. University of Pittsburgh Press, p. 35. Un examen de cuáles son los factores más asociados con la confianza interpersonal encuentran que es la escolaridad de las personas la que mejor predice la inclinación a confiar en los demás (p. 59). Sobre la relación entre escolaridad y confianza interpersonal, Robert Putnam ha encontrado que en Estados Unidos hay un impacto superior de la escolaridad secundaria en la confianza, es decir la escolaridad entre los 14 y 18 años tiene un impacto sobre la confianza interpersonal 10 veces superior a la escolaridad básica, en los países de América Latina incluidos en este estudio, Chile, Costa Rica y México se observa una relación similar en Chile, país en el que el mayor número de personas tienen acceso a la educación secundaria (p. 61).
}

los niños había estado matriculados alguna vez en la escuela, como puede verse en el cuadro 1 . Sin embargo, esta relativa igualdad en la oportunidad inicial de matricularse en la escuela, no resulta en iguales oportunidades de permanecer en la escuela durante el tiempo suficiente para culminar sexto grado, o para culminar la educación básica de nueve grados. A la edad de 12 años seguían matriculados en la escuela todos los niños cuyas familias se encontraban en el $20 \%$ con más ingresos en la población, en comparación con el $86 \%$ de los niños cuyas familias se encontraban entre el $40 \%$ con menos ingresos en la población, por haber desertado el $14 \%$ del $96 \%$ que inició su escolaridad alguna más ingresos han aprobado seis años de escolaridad primaria, sólo la mitad de los niños en el $40 \%$ con menos ingresos han aprobado seis años. A la misma edad, mientras que el $73 \%$ de los niños en el $20 \%$ de las familias con más ingresos han aprobado nueve años de escolaridad básica, sólo uno de cada cuatro niños en el $40 \%$ de las familias con menos ingresos han aprobado ese nivel de escolaridad básica.

Cuadro 1. Probabilidad de estar matriculado a varios niveles y de haber alcanzado sexto y noveno grado a la edad de 15-19 para distintos grupos de ingreso en Colombia, en el año 2000.

\begin{tabular}{|c|c|c|c|}
\hline $\begin{array}{c}\text { Más } \\
\text { ingresos } \\
20 \%\end{array}$ & $\begin{array}{c}40 \% \\
\text { de ingresos } \\
\text { medios }\end{array}$ & $\begin{array}{c}\text { Menores } \\
\text { ingresos } \\
40 \%\end{array}$ & $\begin{array}{c}20 \% \\
\text { más rico/ } \\
40 \% \text { más } \\
\text { pobre }\end{array}$ \\
\hline \multicolumn{4}{|c|}{$\begin{array}{l}\text { Proporción de quienes estuvieron matricula- } \\
\text { dos alguna vez entre los que tienen } 12 \text { años }\end{array}$} \\
\hline 0.99 & 0.99 & 0.96 & $103 \%$ \\
\hline \multicolumn{4}{|c|}{$\begin{array}{l}\text { Proporción de quienes están matriculados en } \\
\text { la escuela a la edad de } 12 \text { años }\end{array}$} \\
\hline 0.99 & 0.95 & 0.83 & $119 \%$ \\
\hline \multicolumn{4}{|c|}{$\begin{array}{l}\text { Proporción de quienes han aprobado sexto } \\
\text { grado (edad } 15 \text { a } 19 \text { años) }\end{array}$} \\
\hline 0.92 & 0.88 & 0.52 & $177 \%$ \\
\hline \multicolumn{4}{|c|}{$\begin{array}{l}\text { Proporción de quienes han aprobado noveno } \\
\text { grado (edad } 15 \text { a } 19 \text { años) }\end{array}$} \\
\hline 0.73 & 0.61 & 0.25 & $292 \%$ \\
\hline
\end{tabular}

Fuente:World Bank. Educational Attainment and Enrollment Around the World.

http://www.worldbank.org/research/projects/edattain/ edattain.htm (accesada 11 de junio de 2002). 
El problema de las altas tasas de deserción escolar entre los grupos más pobres se ve agravado porque la deserción escolar ha estado aumentando en Colombia en los últimos años, lo cual sugiere que las brechas educativas entre grupos sociales aumentaran y que la escolaridad promedio de la población disminuirá aún más en los años venideros, aumentando todavía más las brechas con los países de la OECD y las brechas educativas entre diferentes grupos sociales, y probablemente debilitando aún más las competencias para ejercer ciudadanía asociadas con altos niveles de escolaridad. Es fundamental llevar adelante estudios que permitan conocer quiénes son los estudiantes que desertan de la escuela -ya es sabido que son fundamentalmente los más pobres, pero no todos los pobres desertan- y por qué lo hacen.

Si bien es posible que haya factores extra-escolares que incidan en la deserción (la violencia política que obliga a las familias a desplazarse, el deterioro de la situación económica de las familias, tal vez la percepción de que no hay opciones de empleo que justifiquen el esfuerzo de alcanzar niveles superiores de educación), es fundamental examinar cuál es la responsabilidad de la escuela por la deserción y buscar opciones de intervención allí donde ellas están más al alcance de la acción publica, es decir, en el sistema educativo. Decir el sistema educativo, sin embargo, es llevar el nivel de análisis más allá de la escuela específica que un niño abandona, pues hay circunstancias donde no es posible desde las escuelas que los niños abandonan incidir en las causas del problema. Así, por ejemplo, cuando una familia abandona su lugar de residencia porque las condiciones de violencia en esa zona la obliga a ello, hay poco que las escuelas mismas que van perdiendo a sus estudiantes podrían hacer para retenerlos, pues no pueden ellas resolver el problema de la violencia. Lo que sí puede el sistema educativo hacer es plantearse cómo atender las necesidades educativas de los niños que se insertan como desplazados en nuevas comunidades; por ejemplo, flexibilizando las normas de inscripción para permitir que niños desplazados se matriculen en cualquier momento del año, creando programas de transición y de apoyo en la escuela para desplazados y coordinando con otras instituciones locales la provisión de servicios de salud mental de los niños desplazados y de sus familias.

Desde el punto de vista de contribuir a la democracia, a la estabilidad política y a la paz, hay varias razones de peso para interesarse en los niños que se van de la escuela antes de completar su educación fundamental. Una es que quienes se van proceden fundamentalmente de los sectores más pobres de la sociedad; $y$ al hacerlo pierden la principal oportunidad de las familias de mejorar de una generación a otra sus opciones de vida. Uno de los más claros rasgos que diferencian hoy en día en Colombia a grupos con distintos perfiles de ingreso son sus distintos niveles educativos. En la medida en que los hijos de los pobres abandonen la escuela antes que los demás esto contribuirá a reproducir el círculo vicioso de la pobreza 5 . Una segunda razón es que la desincorporacion de los niños de la escuela, sin competencias que permitan opciones laborales bien remuneradas, y sin ocupación, aumentan la vulnerabilidad de estos niños, convirtiéndolos en potenciales instrumentos para la perpetuación de la violencia en sus diversas manifestaciones. Los jóvenes desocupados

\footnotetext{
${ }^{5}$ En 1997 el 10\% mas pobre de la población en edad laboral había aprobado 3 años de escolaridad, comparado con 12 años de escolaridad entre el $10 \%$ de la población con más ingresos. El 30\% más pobre tenía menos de 5 años de escolaridad aprobada y el $30 \%$ con mas ingresos había aprobado al menos 8 años de escolaridad (Sarmiento Gómez, Alfredo. 2002. "Política y Equidad Educativas en Colombia". En Reimers, F. Ed. Distintas escuelas, diferentes oportunidades. Madrid: Editorial Arco. La Muralla, pp. 327387.
}

y aburridos pueden encontrar estimulación y sentido para sus vidas en actividades al margen de la ley. Una tercera razón es que al abandonar la escuela en grandes números se reducen las posibilidades agregadas del país de generar los bienes y servicios que permitan mejorar las condiciones de vida de toda la población. Finalmente, es el abandono de la escuela, antes que la falta de acceso inicial a ella, la causa principal de que la población más pobre no tenga niveles más altos de escolaridad. El enorme esfuerzo que ha representado para el Estado y para las familias el desarrollo de una cultura que valore la educación lo suficiente para aceptar y esperar que todos los niños sean matriculados en el primer grado de educación primaria, se ve minado cuando los niños abandonan la escuela, en particular antes de culminar la escolarización básica. El aumento reciente de los niveles de deserción es una señal alarmante de que una cultura alternativa, un nuevo conjunto de significados compartidos empieza a instalarse entre amplios grupos de la población y concretamente entre los niños que abandonan la escuela, donde el no terminar los estudios de primaria o el nivel de la educación básica aparecen como opciones de vida cada vez más aceptables.

Un reciente estudio realizado por Sarmiento, Tovar y Alam, muestra claramente cómo el porcentaje de niños que llegan a quinto grado en Colombia $(73 \%)$ es inferior al de otros países con índices de desarrollo humano comparables, incluidos México ( $86 \%$ ) y Venezuela $(89 \%)^{6}$. El mismo estudio confirma que son los niños más pobres quienes, como resultado de la deserción temprana, tienen menos probabilidad de acceder a la educación secundaria. Entre los 7 y los 11 años

\footnotetext{
${ }^{6}$ Sarmiento-Gómez, A.; L. Tovar y C. Alam. 2002. Situación de la Educación Básica, Media y Superior. Casa Editoral El Tiempo, p. 44 .
} 
el $93 \%$ de los niños asisten a la escuela, para el $10 \%$ más pobre solo el $88 \%$, mientras que para el $10 \%$ con más ingresos el $98 \%$ asiste. Entre los 12 y los 17 años, el porcentaje que asiste a la escuela cae a $60 \%$ para el $10 \%$ más pobre, frente a $84 \%$ para el $10 \%$ con más ingresos. Entre los 18 y los 25 años sólo el $11 \%$ del $10 \%$ más pobre asiste a la escuela, comparado con el $27 \%$ entre el $10 \%$ con más ingresos $^{7}$. En síntesis, es claro que quienes se van de la escuela son los más pobres y que con ello las brechas de oportunidad educativa crecen exponencialmente. Las brechas de oportunidad de estar estudiando entre ricos y pobres, de $10 \%$ entre los 7 y 11 años, son tres veces mayores entre los 12 y los 17 años, y seis veces mayores entre los 18 $y$ los 25 años.

Un hallazgo fundamental y de gravísimas implicaciones del estudio anteriormente mencionado es que la proporción de estudiantes que asisten a la escuela ha comenzado a disminuir para los grupos más pobres a partir de 1997. Por ejemplo, entre los 7 y 11 años, la asistencia escolar para el $10 \%$ más pobre disminuye de $92 \%$ en 1997 a $88 \%$ en 2000 , para el siguiente $10 \%$ de ingreso de $94 \%$ a $90 \%$, para el siguiente $10 \%$ disminuyó de $97 \%$ a $89 \%$. En todos los demás grupos de ingreso la asistencia escolar aumentó. Como resultado, las brechas de oportunidad mencionadas están aumentando.

Disminuciones aún más dramáticas se observan en la asistencia escolar de los cuatro deciles más pobres, entre 12 y 17 años, mientras que la asistencia escolar aumenta para todos los demás deciles de ingreso. Para el decíl más pobre la asistencia de quienes tienen entre 12 y 17 años cae de $69 \%$ en 1997 a $60 \%$ en el 2000, para el siguiente decil cae del $73 \%$ al $59 \%$, para el siguien-

\footnotetext{
7 Ibíd., p. 32.
}

te decil del $84 \%$ al $65 \%$, para el siguiente decil, de $80 \%$ a $66 \%$. Es decir, a partir de 1997 los hijos del 30-40\% más pobre del país pierden oportunidades de acceso a la educación que ya habían alcanzado, ello mientras los hijos de los restantes grupos de ingreso aumentan sus tasas de asistencia escolar. El porcentaje de asistencia es ligeramente menor entre los niños que entre las niñas para el grupo de 12 a 17 años $(75 \%$ vs. $77 \%)$.

Es posible que estos aumentos de la deserción escolar se deban a la profundización de la recesión económica, también podrían deberse a desplazamientos resultantes de la escalada de violencia política. Según datos de CODHES, se desplazan actualmente 42 personas cada hora por causa de la violencia ${ }^{8}$. La disrupción, al menos temporal, que estos desplazamientos causan en las oportunidades de estudio de los hijos de estas familias desplazadas es obvia. Es posible, además, que una vez interrumpidas las trayectorias educativas de estos estudiantes y como consecuencia de las múltiples vulnerabilidades de las familias desplazadas, esta deserción de la escuela sea permanente.

Considerando la evidencia existente en otros contextos que sugiere que la deserción es un proceso multicausado y que a ella contribuye la baja calidad de la oferta educativa que resulta inicialmente en repitencia y eventualmente en la expulsión de la escuela, es posible también que la deserción en Colombia -la más nueva expresada en los aumentos a partir de 1997 mencionados, así como los niveles preexistentes de deserción- resulte en parte de la baja calidad de la oferta educativa. Los niños y sus familias toman la decisión de abandonar la escuela, en parte porque no perciben que los beneficios que ella les ofrece justifican los enormes esfuerzos que asistir representa.

\footnotetext{
8 www.codhes.org.co
}

El mismo estudio mencionado muestra que las más altas tasas de deserción ocurren en primer grado de primaria (18\%) y en sexto grado de secundaria $(15 \%)$. Las tasas de deserción son significativamente más altas en las escuelas rurales que en las urbanas y en las escuelas oficiales que en las privadas. Los autores de este estudio atribuyen a la crisis económica el significativo aumento de las tasas de deserción en escuelas privadas y el aumento de las tasas de deserción en escuelas oficiales secundarias, a partir de 1997.

Analizando los resultados de la encuesta de condiciones de vida realizada en 1997, este estudio encuentra que la principal causa a la que las familias atribuyen que sus hijos no estén en la escuela son los costos que para las familias tiene la educación de sus hijos (44\% de los estudiantes entre 7 y 11 años y $35 \%$ de aquellos entre 12 y 17 años) seguida por "No le gusta" (14\% de los estudiantes de 7 a 11 años y $29 \%$ de aquellos entre 12 y 17 años).

Estos resultados sugieren que las principales áreas sobre las que se podría incidir en los niveles de deserción (sobre la base de información proporcionada por las familias en 1997) serían los costos y la falta de atractivo de la educación secundaria para algunos de los jóvenes. Es posible que los mismos factores expliquen el aumento de la deserción a partir de 1997 como resultado de la acentuación de la crisis económica. Pero también es posible que nuevos factores expliquen la "nueva deserción" en particular en los primeros grados de primaria.

Avanzar en reducir la proporción de los estudiantes que se van requerirá avanzar en la comprensión del problema, en la identificación de opciones de acción y en la implementación de cambios en la escuela y en el sistema educativo. 
Segundo desafío. Los estudiantes en las escuelas rurales siguen aprendiendo muy poco. La prioridad de la lecto-escritura

Los niños en las escuelas rurales en América Latina siguen aprendiendo muy poco. Hay brechas con escuelas urbanas y privadas y muy pocas diferencias entre los países de América Latina. Utilizando los resultados del estudio llevado a cabo por los Ministerios de Educación de varios países de América Latina, coordinados por el Laboratorio de la Calidad de la Educación de la Oficina Regional de UNESCO en Santiago a fines de la década pasada, es posible ver que los niños aprenden muy poquito, alcanzando apenas un $50 \%$ de los objetivos del currículo de lengua. Si los niños no conocen el idioma, si leen con dificultad, es difícil que puedan aprender por su cuenta, que puedan apoyarse en materiales de auto instrucción o que puedan seguir aprendiendo a lo largo de su vida.

Cuadro 2. Resultados Promedio en la prueba de Lenguaje de estudiantes de cuarto grado en varios países de América Latina en 1998.

\begin{tabular}{|c|c|c|c|}
\hline País & Rural & Pública & Privada \\
\hline Argentina & 12.49 & 14.37 & 16.70 \\
\hline Bolivia & 9.39 & 10.84 & 13.99 \\
\hline Brasil & 12.37 & 14.03 & 15.34 \\
\hline Chile & 12.86 & 12.46 & 15.17 \\
\hline Colombia & 12.07 & 13.10 & 14.51 \\
\hline Cuba & 16.70 & 17.60 & \\
\hline Honduras & 9.69 & 11.81 & 13.66 \\
\hline México & 10.97 & 12.79 & 15.56 \\
\hline Paraguay & 11.08 & & \\
\hline Perú & 9.21 & 11.71 & 14.43 \\
\hline \multicolumn{4}{|l|}{ Rep. } \\
\hline Dominicana & 9.25 & 11.28 & 12.04 \\
\hline Venezuela & 11.66 & 12.76 & 12.81 \\
\hline Total & 11.31 & 13.27 & 14.21 \\
\hline
\end{tabular}

Fuente:Procesamiento propio sobre la base de datos construida por UNESCO-OREALC. Laboratorio de la Calidad de la Educación en base a encuestas y pruebas administradas por los ministerios de educación hacia 1998. Las dos últimas columnas se refieren sólo a las ciudades de más de un millón de habitantes.
A pesar de que el número de personas analfabetas reportado en el Censo es inferior al $10 \%$, en un reciente estudio de competencias lectoras de estudiantes de cuarto grado, llevado a cabo en 31 países, las capacidades lectoras de los estudiantes de Colombia estuvieron entre las más bajas en el mundo: menos de $25 \%$ de los estudiantes colombianos obtuvieron resultados superiores al promedio internacional, y sólo 4 países (Irán, Kuwait, Marruecos y Belice) tuvieron resultados promedio más bajos (Progress in International Reading Literacy Study, 2001).

Con debilidades en la comprensión lectora es difícil aprender otras asignaturas. Estos bajos niveles de habilidades cognoscitivas de los estudiantes colombianos son confirmados por el Tercer Estudio de Matemáticas y Ciencias (TIMSS). Este estudio, que evaluó el desempeño académico de estudiantes en 39 países del mundo en séptimo y octavo grados a comienzos de los noventa muestra que sólo los estudiantes de Sudáfrica, con su larga historia de discriminación racial expresada en bajísimas oportunidades educativas en las escuelas para los estudiantes negros, tienen niveles de desempeño ligeramente más bajos que los estudiantes de Colombia. En una escala de 800 puntos los estudiantes colombianos de séptimo grado obtuvieron en promedio 369 puntos en matemáticas, comparados con un promedio en el conjunto de los 39 países de 484 puntos, y con un promedio de 476 puntos en Estados Unidos y de 494 puntos en Canadá. Los resultados en la prueba de ciencias de octavo grado fueron iguales de bajos, promediando 411 puntos entre los estudiantes

\footnotetext{
${ }^{9}$ Beaton, A., I. Mullis, M. Martin, E. González, D. Kelly and T. Smith. 1996. Mathematics Achievement in the Middle school Years: IEA's Third International Mathematics and Science Study (TIMSS). International Association for the Evaluation of Educational Achievement. Chestnut Hill, MA. Boston College, p. 23.
}

colombianos, con relación a un promedio de 516 para el conjunto de los países. De nuevo sólo los estudiantes de Sudáfrica obtuvieron puntajes inferiores a los estudiantes colombianos ${ }^{10}$.

El desarrollo de las habilidades de lecto-escritura debe ser una prioridad fundamental en el fortalecimiento de las escuelas rurales en América Latina. Los problemas empiezan desde que comienza la escuela, probablemente antes, como lo reflejan las altísimas tasas de repitencia escolar en el primer grado, un tema cuyo reconocimiento se debe a la persistente labor de investigación y difusión del gran educador latinoamericano Ernesto Schiefelbein. Como muestra, un porcentaje muy alto de los estudiantes de cuarto grado han repetido primer grado por lo menos una vez.

Cuadro 3. Porcentaje de Estudiantes en Escuelas Rurales en cuarto grado que han repetido al menos una vez primero, segundo y tercer grado.

\begin{tabular}{|lrrr|}
\hline País & Primero & Segundo & Tercero \\
\hline Argentina & $20 \%$ & $7 \%$ & $3 \%$ \\
Bolivia & $19 \%$ & $13 \%$ & $10 \%$ \\
Brasil & $24 \%$ & $13 \%$ & $13 \%$ \\
Chile & $7 \%$ & $6 \%$ & $5 \%$ \\
Colombia & $17 \%$ & $7 \%$ & $4 \%$ \\
Cuba & $0 \%$ & $2 \%$ & $0 \%$ \\
Honduras & $20 \%$ & $7 \%$ & $20 \%$ \\
México & $29 \%$ & $25 \%$ & $24 \%$ \\
Paraguay & $23 \%$ & $20 \%$ & $9 \%$ \\
Perú & $22 \%$ & $10 \%$ & $9 \%$ \\
Rep. & & & \\
Dominicana & $17 \%$ & $10 \%$ & $13 \%$ \\
Venezuela & $18 \%$ & $11 \%$ & $8 \%$ \\
\hline
\end{tabular}

Fuente: Procesamiento propio sobre la base de datos construida por UNESCO-OREALC. Laboratorio de la Calidad de la Educación en base a encuestas y pruebas administradas por los ministerios de educación hacia 1998.

\begin{abstract}
${ }^{10}$ Beaton, A., I. Mullis, M. Martin, E. González, D. Kelly and T. Smith. 1996. Science Achievement in the Middle school Years: IEA's Third International Mathematics and Science Study (TIMSS). International Association for the Evaluation of Educational Achievement. Chestnut Hill, MA. Boston College, p. 23.
\end{abstract}




\section{La explicación de los bajos niveles de aprendizaje: pedagogía inefectiva e insuficientes materiales de lectura}

Hay tres razones por las cuales los estudiantes en escuelas rurales en América Latina tienen dificultad para dominar destrezas de lectura y comunicación, y probablemente otros conocimientos y disposiciones. Una es que las pedagogías de sus profesores son deficientes. Otra es que los estudiantes y los profesores tienen muy pocos materiales a su disposición para apoyar su aprendizaje. Una tercera es que la cultura de la escuela no está centrada en apoyar el éxito escolar de los niños y el énfasis en la enseñanza efectiva. Los equipos docentes en las escuelas tienen poca experiencia en trabajar en equipo para establecer metas, para definir programas de autoformación, para estudiar las causas de los problemas de sus estudiantes.

\section{Pedagogía inefectiva}

Al preguntársele a los niños, en la encuesta mencionada anteriormente, si entendían las explicaciones de sus maestros, sólo la mitad dijeron que entendía todo lo que explicaban sus maestros. Para contextualizar la gravedad de esta afirmación, imaginémonos qué sería de otras profesiones si la mitad de los clientes de los servicios de estos profesionales dijeran no estar satisfechos. ¿Qué pensaríamos de un médico si sólo la mitad de sus pacientes dijesen que los servicios de éste médico les han ayudado a recuperar la salud? ¿Qué pensaríamos de un piloto de avión si su record fuese el de llevar a sus pasajeros a su destino exitosamente solo en la mitad de sus vuelos? ¿Qué pensaríamos de un cocinero cuyas comidas le cayesen bien a sus comensales solo la mitad de las veces? La enseñanza en América Latina está en crisis a juzgar por los pobres resultados de aprendizaje. En especial es necesario ayudar a los profesores a desarrollar competencias que les permitan dominar los contenidos que enseñan, a desarrollar pedagogías altamente efectivas para enseñar diferentes asignaturas, a obtener conocimiento sobre sus estudiantes. A estos temas me referiré más adelante.

Cuadro 4. Porcentaje de alumnos de cuarto grado en escuelas rurales que indican que entienden las explicaciones de sus profesores.

\begin{tabular}{|lccc|}
\hline País & Todo & $\begin{array}{c}\text { Algunas } \\
\text { cosas }\end{array}$ & Nada \\
\hline Argentina & $56 \%$ & $43 \%$ & $1 \%$ \\
Bolivia & $60 \%$ & $37 \%$ & $3 \%$ \\
Brasil & $51 \%$ & $48 \%$ & $1 \%$ \\
Chile & $48 \%$ & $49 \%$ & $2 \%$ \\
Colombia & $51 \%$ & $48 \%$ & $1 \%$ \\
Cuba & $88 \%$ & $11 \%$ & $0 \%$ \\
Honduras & $68 \%$ & $29 \%$ & $2 \%$ \\
México & $59 \%$ & $40 \%$ & $1 \%$ \\
Paraguay & $64 \%$ & $32 \%$ & $3 \%$ \\
Perú & $55 \%$ & $43 \%$ & $2 \%$ \\
Rep. & & & \\
Dominicana & $64 \%$ & $35 \%$ & $2 \%$ \\
Venezuela & $59 \%$ & $40 \%$ & $1 \%$ \\
\hline
\end{tabular}

Fuente: Procesamiento propio sobre la base de datos construida por UNESCO-OREALC. Laboratorio de la Calidad de la Educación con base en encuestas y pruebas administradas por los Ministerios de Educación, hacia 1998.

El porcentaje de estudiantes que entienden todo lo que explica el maestro es menor en escuelas rurales que en las demás, como muestra el cuadro 5, para el caso de Colombia.

Cuadro 5. Porcentaje de estudiantes de tercero y cuarto grados que entienden lo que el maestro explica en distintos tipos de escuela en 1998 en Colombia.

\begin{tabular}{|c|c|c|c|}
\hline País & Todo & $\begin{array}{c}\text { Algunas } \\
\text { cosas }\end{array}$ & Nada \\
\hline Mega-Público & $61.8 \%$ & $35.8 \%$ & $2.4 \%$ \\
\hline Mega-Privado & $62.0 \%$ & $37.7 \%$ & $.4 \%$ \\
\hline Urbano-Público & $61.9 \%$ & $36.3 \%$ & $1.8 \%$ \\
\hline Urbano-Privado & $64.1 \%$ & $35.5 \%$ & $.4 \%$ \\
\hline Rural & $54.3 \%$ & $45.2 \%$ & $.5 \%$ \\
\hline Total & $60.0 \%$ & $38.8 \%$ & $1.2 \%$ \\
\hline
\end{tabular}

Fuente: Cálculos propios de datos procedentes de la encuesta administrada por el Ministerio de Educación y la Oficina Regional de UNESCO para América Latina y el Caribe (Laboratorio Latinoamericano de la Calidad de la Educación). Estrato Mega Ciudad se refiere a ciudades con más de 1 millón de habitantes. Urbano se refiere a ciudades de menos de 1 millón de habitantes.
La forma en que las maestras explican está, pues, fuera del alcance de $40 \%$ de los niños. Lo que es más grave, aquellos que menos entienden están menos inclinados a pedir ayuda adicional al maestro. Al preguntarle a los niños qué hacen cuando tienen dudas con una explicación, entre quienes entienden todo lo que el maestro explica un porcentaje mayor pregunta de inmediato al maestro, entre quienes no entienden nada, muy pocos preguntan de inmediato al maestro. Una proporción mucho mayor de los niños que no entienden las explicaciones de los profesores se apoya en sus compañeros o no hace nada.

Cuadro 6. Porcentaje de estudiantes de tercero y cuarto grados que piden aclaración de sus dudas por cuanto entienden las explicaciones de los maestros en 1998.

\begin{tabular}{|lrrrr|}
\hline $\begin{array}{l}\text { En caso } \\
\text { de duda }\end{array}$ & Todo & $\begin{array}{c}\text { Algunas } \\
\text { cosas }\end{array}$ & Nada & Total \\
\hline $\begin{array}{l}\text { Preguntade } \\
\text { inmediato }\end{array}$ & $75.7 \%$ & $64.0 \%$ & $26.7 \%$ & $70.7 \%$ \\
$\begin{array}{l}\text { Pregunta después } \\
\text { Espera siguiente }\end{array}$ & $10.1 \%$ & $10.7 \%$ & $22.2 \%$ & $10.5 \%$ \\
lase & $4.7 \%$ & $8.4 \%$ & $22.2 \%$ & $6.3 \%$ \\
$\begin{array}{l}\text { Pregunta a otros } \\
\text { No pregunta }\end{array}$ & $5.5 \%$ & $11.4 \%$ & $11.1 \%$ & $7.8 \%$ \\
\hline Total & $3.9 \%$ & $5.5 \%$ & $17.8 \%$ & $4.7 \%$ \\
\hline
\end{tabular}

Fuente:Cálculos propios de datos procedentes de la encuesta administrada por el Ministerio de Educación y la Oficina Regional de UNESCO para América Latina y el Caribe (Laboratorio Latinoamericano de la Calidad de la Educación). Estrato Mega Ciudad se refiere a ciudades con más de 1 millón de habitantes. Urbano se refiere a ciudades de menos de 1 millón de habitantes.

Aparte de que un buen número de estudiantes no entiende las explicaciones de los profesores, las lecturas que hacen los maestros no son estimulantes para los estudiantes.

Al preguntarles qué es lo que más les gustaba de la escuela sólo $17 \%$ mencionaron como primera opción las lecturas de los profesores, y un $8 \%$ adicional mencionaron aprender, mientras que un $67 \%$ mencionaron el deporte. Como segunda opción, un porcentaje mayor menciona aprender (46\%), pero tan solo $14 \%$ mencionan las lecturas del maestro como lo que más les gusta. 
Cuadro 7. Porcentaje de estudiantes de tercero y cuarto grado que indican cuales son las actividades que mas les gustan en la escuela.

\begin{tabular}{|c|c|c|c|c|c|}
\hline Primera opción & Deporte & $\begin{array}{c}\text { Lecturas } \\
\text { del } \\
\text { maestro }\end{array}$ & Jugar & Aprender & Vacaciones \\
\hline Mega-Público & $64.3 \%$ & $16.7 \%$ & $9.6 \%$ & $8.3 \%$ & $1.1 \%$ \\
\hline Mega-Privado & $67.6 \%$ & $15.7 \%$ & $7.8 \%$ & $8.3 \%$ & $.6 \%$ \\
\hline Urbano-Público & $66.5 \%$ & $16.1 \%$ & $7.3 \%$ & $8.8 \%$ & $1.3 \%$ \\
\hline Urbano-Privado & $67.5 \%$ & $13.7 \%$ & $8.7 \%$ & $9.1 \%$ & $1.0 \%$ \\
\hline Rural & $67.5 \%$ & $19.9 \%$ & $6.5 \%$ & $6.1 \%$ & $.1 \%$ \\
\hline Total & $66.8 \%$ & $16.9 \%$ & $7.6 \%$ & $7.9 \%$ & $.8 \%$ \\
\hline Primera opción & Deporte & $\begin{array}{c}\text { Lecturas } \\
\text { del } \\
\text { maestro }\end{array}$ & Jugar & Aprender & Vacaciones \\
\hline Mega-Público & $.7 \%$ & $11.3 \%$ & $13.4 \%$ & $41.1 \%$ & $33.6 \%$ \\
\hline Mega-Privado & $.8 \%$ & $10.5 \%$ & $12.2 \%$ & $44.8 \%$ & $31.6 \%$ \\
\hline Urbano-Público & $.3 \%$ & $17.2 \%$ & $13.5 \%$ & $45.5 \%$ & $23.5 \%$ \\
\hline Urbano-Privado & $.4 \%$ & $9.4 \%$ & $10.7 \%$ & $41.1 \%$ & $38.3 \%$ \\
\hline Rural & $.4 \%$ & $13.9 \%$ & $11.8 \%$ & $52.0 \%$ & $22.0 \%$ \\
\hline Total & $.5 \%$ & $13.7 \%$ & $12.5 \%$ & $46.3 \%$ & $27.1 \%$ \\
\hline
\end{tabular}

Fuente: Cálculos propios de datos procedentes de la encuesta administrada por el Ministerio de Educación y la Oficina Regional de UNESCO para América Latina y el Caribe (Laboratorio Latinoamericano de la Calidad de la Educación). Estrato Mega Ciudad se refiere a ciudades con más de 1 millón de habitantes. Urbano se refiere a ciudades de menos de 1 millón de habitantes.

Las dificultades para desarrollar buenas competencias lectoras que resultan de las deficientes explicaciones de los profesores y de lo poco estimulante de las lecturas que los profesores hacen, se ven agudizadas por la carencia que tienen algunos estudiantes de materiales básicos de aprendizaje, así como por las limitadas oportunidades que tienen de leer fuera de la escuela.

\section{Insuficientes materiales}

En parte la deficiente capacidad pedagógica de los profesores resulta de su deficiente formación y de las insuficientes oportunidades efectivas de actualización. En parte resulta también de la carencia de condiciones básicas en las escuelas rurales. La misma encuesta referida anteriormente muestra cómo hay aún niños que no tienen libros de texto, cuadernos o lápices. Es este segundo desafió, la creación de contextos de aprendizaje ricos en materiales que los niños puedan utilizar, uno que debe ser atendido prioritariamente. Es esta una de las fortalezas del modelos de Escuela Nueva, al menos en su formulación original, el enfatizar las guías de aprendizaje, las bibliotecas de aula, y los materiales pedagógicos para apoyar la labor del maestro.

Cuadro 8. Porcentaje de alumnos de cuarto grado en escuelas rurales que indican que tienen materiales básicos de aprendizaje.

\begin{tabular}{|c|c|c|c|}
\hline País & $\begin{array}{c}\text { Libro } \\
\text { del } \\
\text { lenguaje }\end{array}$ & $\begin{array}{l}\text { Cua- } \\
\text { dernos }\end{array}$ & $\begin{array}{c}\text { Lápi- } \\
\text { ces }\end{array}$ \\
\hline Argentina & $64 \%$ & $82 \%$ & $88 \%$ \\
\hline Bolivia & $37 \%$ & $82 \%$ & $90 \%$ \\
\hline Brasil & $94 \%$ & $97 \%$ & $94 \%$ \\
\hline Chile & $91 \%$ & $88 \%$ & $91 \%$ \\
\hline Colombia & $67 \%$ & $90 \%$ & $94 \%$ \\
\hline Cuba & $98 \%$ & $99 \%$ & $99 \%$ \\
\hline Honduras & $70 \%$ & $84 \%$ & $90 \%$ \\
\hline México & $96 \%$ & $97 \%$ & $96 \%$ \\
\hline Paraguay & $71 \%$ & $83 \%$ & $80 \%$ \\
\hline Perú & $44 \%$ & $74 \%$ & $84 \%$ \\
\hline Rep. & & & \\
\hline Dominicana & $51 \%$ & $77 \%$ & $85 \%$ \\
\hline Venezuela & $63 \%$ & $83 \%$ & $83 \%$ \\
\hline
\end{tabular}

Fuente: Procesamiento propio sobre la base de datos construida por UNESCO-OREALC. Laboratorio de la Calidad de la Educación con base en encuestas y pruebas administradas por los ministerios de educación hacia 1998.

Treinta por ciento de los alumnos no tienen libros de lenguaje, $12 \%$ no tienen cuadernos y $8 \%$ no tienen ni siquiera un lápiz con qué escribir. Curiosamente, la carencia de materiales de aprendizaje no es superior en zonas rurales en Colombia que en zonas urbanas como muestra el siguiente cuadro.
Cuadro 9. Porcentaje de niños de tercero y cuarto grado que tienen materiales básicos de aprendizaje.

\begin{tabular}{|lcccc|}
\hline & $\begin{array}{c}\text { Libro } \\
\text { de } \\
\text { Len- } \\
\text { guaje }\end{array}$ & $\begin{array}{c}\text { Libro } \\
\text { de } \\
\text { Mate- } \\
\text { máticas }\end{array}$ & $\begin{array}{c}\text { Cua- } \\
\text { dernos }\end{array}$ & $\begin{array}{c}\text { Lápi- } \\
\text { ces }\end{array}$ \\
\hline Mega-Público & $70 \%$ & $59 \%$ & $88 \%$ & $93 \%$ \\
Mega-Privado & $79 \%$ & $70 \%$ & $89 \%$ & $90 \%$ \\
Urbano-Público & $67 \%$ & $54 \%$ & $85 \%$ & $91 \%$ \\
Urbano-Privado & $84 \%$ & $78 \%$ & $94 \%$ & $93 \%$ \\
Rural & $70 \%$ & $67 \%$ & $88 \%$ & $94 \%$ \\
\hline Total & $72 \%$ & $63 \%$ & $88 \%$ & $92 \%$ \\
\hline
\end{tabular}

Fuente:Cálculos propios de datos procedentes de la encuesta administrada por el Ministerio de Educación y la Oficina Regional de UNESCO para América Latina y el Caribe (Laboratorio Latinoamericano de la Calidad de la Educación). Estrato Mega Ciudad se refiere a ciudades con más de 1 millón de habitantes. Urbano se refiere a ciudades de menos de 1 millón de habitantes.

La falta de acceso a materiales básicos de lectura es confirmada por las respuestas de los profesores a la pregunta de si alcanzan los textos para cada niño, a la cual 2 de cada cinco maestros dice que no.

Cuadro 10. Respuestas de los maestros a la pregunta ¿Alcanzan para cada niño los textos?

\begin{tabular}{|lcc|}
\hline & No & Si \\
\hline Mega-Público & $34.5 \%$ & $65.5 \%$ \\
Mega-Privado & $23.5 \%$ & $76.5 \%$ \\
Urbano-Público & $45.7 \%$ & $54.3 \%$ \\
Urbano-Privado & $32.1 \%$ & $67.9 \%$ \\
Rural & $49.3 \%$ & $50.7 \%$ \\
\hline Total & $41.4 \%$ & $58.6 \%$ \\
\hline
\end{tabular}

Fuente:Cálculos propios de datos procedentes de la encuesta administrada por el Ministerio de Educación y la Oficina Regional de UNESCO para América Latina y el Caribe (Laboratorio Latinoamericano de la Calidad de la Educación). Estrato Mega Ciudad se refiere a ciudades con más de 1 millón de habitantes. Urbano se refiere a ciudades de menos de 1 millón de habitantes.

La carencia de materiales básicos de aprendizaje impide la práctica regular de la lectura. Al preguntar a los estudiantes qué habían leído la semana anterior a la encuesta, sólo 3 de cada cinco indicaron que habían leído un libro, casi la mitad que habían leído comics y dos de cada cinco que había leído periódicos. Leer a otras personas o en voz alta es una práctica infrecuente. Casi $15 \%$ dijeron que no habían leído nada. 
Cuadro 11. Porcentaje de niños de tercero y cuarto grados que indica que realizo diversos tipos de lectura la semana anterior a la encuesta en Colombia.

\begin{tabular}{|lccccccc|}
\hline & Libro & Comics & Periódico & $\begin{array}{c}\text { Leyóa } \\
\text { hermanos }\end{array}$ & $\begin{array}{c}\text { Leyó en } \\
\text { voz alta }\end{array}$ & $\begin{array}{c}\text { Leyó } \\
\text { otra cosa }\end{array}$ & $\begin{array}{c}\text { No } \\
\text { leyó }\end{array}$ \\
\hline Mega-Público & $63 \%$ & $46 \%$ & $36 \%$ & $33 \%$ & $39 \%$ & $31 \%$ & $16 \%$ \\
Mega-Privado & $64 \%$ & $45 \%$ & $32 \%$ & $27 \%$ & $33 \%$ & $30 \%$ & $14 \%$ \\
Urbano-Público & $65 \%$ & $46 \%$ & $43 \%$ & $39 \%$ & $45 \%$ & $35 \%$ & $14 \%$ \\
Urbano-Privado & $63 \%$ & $53 \%$ & $41 \%$ & $33 \%$ & $36 \%$ & $35 \%$ & $15 \%$ \\
Rural & $68 \%$ & $38 \%$ & $44 \%$ & $38 \%$ & $43 \%$ & $38 \%$ & $14 \%$ \\
\hline Total & $65 \%$ & $45 \%$ & $41 \%$ & $36 \%$ & $41 \%$ & $35 \%$ & $14 \%$ \\
\hline
\end{tabular}

Fuente:Cálculos propios de datos procedentes de la encuesta administrada por el Ministerio de Educación y la Oficina Regional de UNESCO para América Latina y el Caribe (Laboratorio Latinoamericano de la Calidad de la Educación). Estrato Mega Ciudad se refiere a ciudades con mas de 1 millón de habitantes. Urbano se refiere a ciudades de menos de 1 millón de habitantes.

Las limitadas oportunidades para leer se relacionan también con la carencia de materiales de lectura en los hogares de los niños y con la infrecuente práctica de que los padres lean a los niños. En casi dos de cada cinco hogares, no hay libros para leer, y en 4 de cada 5 hogares rurales y en 3 de cada 5 hogares de alumnos de escuelas públicas hay menos de 10 libros. Uno de cada cinco niños sus padres no les leían casi nunca, y a otro de cada cinco niños les leían infrecuentemente, menos de 1 vez al mes. Sólo una tercera parte de los niños tienen padres que les leían casi diariamente cuando pequeños. Dado que la ausencia de materiales de lectura en el hogar es muy superior en zonas rurales, la carencia de materiales básicos de lectura en la escuela y de libros de texto en estos ambientes tiene mayor posibilidad de limitar las oportunidades de aprendizaje de lectura entre los estudiantes en estas escuelas que entre los estudiantes que tienen acceso a otros materiales de lectura en el hogar.

Cuadro 12. Cantidad de libros que los estudiantes indican que hay en sus casas.

\begin{tabular}{|lrccc|}
\hline & No hay & Menos de $\mathbf{1 0}$ & De $\mathbf{1 0}$ a $\mathbf{5 0}$ & Más de $\mathbf{5 0}$ \\
\hline Mega-Público & $7.9 \%$ & $38.8 \%$ & $40.7 \%$ & $12.6 \%$ \\
Mega-Privado & $2.6 \%$ & $26.8 \%$ & $43.5 \%$ & $27.1 \%$ \\
Urbano-Público & $20.2 \%$ & $44.8 \%$ & $25.4 \%$ & $9.6 \%$ \\
Urbano-Privado & $3.5 \%$ & $30.6 \%$ & $40.7 \%$ & $25.2 \%$ \\
Rural & $28.7 \%$ & $49.8 \%$ & $17.6 \%$ & $3.9 \%$ \\
\hline Total & $17.4 \%$ & $41.8 \%$ & $28.7 \%$ & $12.2 \%$ \\
\hline
\end{tabular}

Fuente:Cálculos propios de datos procedentes de la encuesta administrada por el Ministerio de Educación y la Oficina Regional de UNESCO para América Latina y el Caribe (Laboratorio Latinoamericano de la Calidad de la Educación). Estrato Mega Ciudad se refiere a ciudades con más de 1 millón de habitantes. Urbano se refiere a ciudades de menos de 1 millón de habitantes.

Cuadro 13. Respuesta de los padres a la pregunta de si acostumbraba a leerle al niño cuando pequeño.

\begin{tabular}{|lccccc|}
\hline & $\begin{array}{c}\text { Casi } \\
\text { diario }\end{array}$ & $\begin{array}{c}\text { Más de 1 vez } \\
\text { al mes }\end{array}$ & $\begin{array}{c}\mathbf{1} \text { vez } \\
\text { al mes }\end{array}$ & $\begin{array}{c}\text { 2 a 3 veces } \\
\text { al año }\end{array}$ & $\begin{array}{c}\text { Casi } \\
\text { nunca }\end{array}$ \\
\hline Mega-Público & $19.1 \%$ & $30.6 \%$ & $10.7 \%$ & $17.7 \%$ & $21.9 \%$ \\
Mega-Privado & $21.9 \%$ & $37.1 \%$ & $11.2 \%$ & $12.8 \%$ & $17.0 \%$ \\
Urbano-Público & $29.6 \%$ & $29.2 \%$ & $10.7 \%$ & $13.8 \%$ & $16.8 \%$ \\
Urbano-Privado & $34.2 \%$ & $36.1 \%$ & $9.1 \%$ & $10.6 \%$ & $10.1 \%$ \\
Rural & $26.0 \%$ & $25.8 \%$ & $13.4 \%$ & $12.7 \%$ & $22.2 \%$ \\
& $26.9 \%$ & $30.1 \%$ & $11.4 \%$ & $13.3 \%$ & $18.2 \%$ \\
\hline
\end{tabular}

Fuente:Cálculos propios de datos procedentes de la encuesta administrada por el Ministerio de Educación y la Oficina Regional de UNESCO para América Latina y el Caribe (Laboratorio Latinoamericano de la Calidad de la Educación). Estrato Mega Ciudad se refiere a ciudades con más de 1 millón de habitantes. Urbano se refiere a ciudades de menos de 1 millón de habitantes.
En síntesis, la pedagogía que utilizan las maestras en las escuelas primarias no es efectiva para que una tercera parte de los estudiantes comprendan. La mayoría de los estudiantes no encuentran estimulantes las lecturas que hacen sus profesores. Esto hace que los ambientes escolares sean débiles para promover el interés por la lectura y el desarrollo de habilidades lectoras efectivas. Esto se ve agravado por la carencia de materiales básicos de lectura en el caso de algunos estudiantes, y por los pocos materiales de lectura que existen en los hogares de muchos estudiantes. Son pocos los estudiantes cuyos padres acostumbraban a leerles cuando pequeños.

\section{Tercer desafío. Deficiente formación para la convivencia democrática y la paz}

Las débiles competencias pedagógicas de los profesores se reflejan no sólo en su incapacidad para desarrollar competencias lectoras en todos los estudiantes, sino en la incapacidad de crear climas instruccionales respetuosos donde los estudiantes adquieran habilidades interpersonales fundamentales para la convivencia democrática. Mencionaba anteriormente que una de las disposiciones esenciales para la vida en democracia es la capacidad de confiar en los demás. Maestros que establecen un clima instruccional respetuoso, que respetan a sus estudiantes y que les enseñan a respetarse unos a otros, contribuyen a que los estudiantes aprendan a confiar en los demás. En la encuesta mencionada anteriormente, por el contrario, un porcentaje elevado de los estudiantes indicaron que no confiaban en sus profesores. 
Cuadro 14. Porcentaje de estudiantes que indican que tiene confianza en el maestro.

\begin{tabular}{|lcccc|}
\hline & Sí & A veces & No & Total \\
\hline Mega-Público & $24.2 \%$ & $38.5 \%$ & $37.2 \%$ & $100.0 \%$ \\
Mega-Privado & $27.0 \%$ & $39.5 \%$ & $33.5 \%$ & $100.0 \%$ \\
Urbano-Público & $29.7 \%$ & $38.2 \%$ & $32.1 \%$ & $100.0 \%$ \\
Urbano-Privado & $27.2 \%$ & $41.1 \%$ & $31.7 \%$ & $100.0 \%$ \\
Rural & $32.0 \%$ & $39.3 \%$ & $28.8 \%$ & $100.0 \%$ \\
\hline Total & $29.1 \%$ & $39.1 \%$ & $31.9 \%$ & $100.0 \%$ \\
\hline
\end{tabular}

Fuente:Cálculos propios de datos procedentes de la encuesta administrada por el Ministerio de Educación y la Oficina Regional de UNESCO para América Latina y el Caribe (Laboratorio Latinoamericano de la Calidad de la Educación). Estrato Mega Ciudad se refiere a ciudades con más de 1 millón de habitantes. Urbano se refiere a ciudades de menos de 1 millón de habitantes.

Igualmente, al preguntar a los estudiantes por sus relaciones con los compañeros, una gran mayoría indican que los compañeros molestan, y una tercera parte indican que tienen peleas constantes con sus

Cuadro 15. Porcentaje de estudiantes que indican que sus compañeros molestan, que tienen peleas constantes y que tienen buenas amistades en la escuela.

\begin{tabular}{|lccc|}
\hline & $\begin{array}{c}\text { Los compañeros } \\
\text { molestan }\end{array}$ & $\begin{array}{c}\text { Peleas } \\
\text { constantes }\end{array}$ & $\begin{array}{c}\text { Buenas } \\
\text { amistades }\end{array}$ \\
\hline Mega-Público & $94 \%$ & $30 \%$ & $90 \%$ \\
Mega-Privado & $89 \%$ & $20 \%$ & $94 \%$ \\
Urbano-Público & $90 \%$ & $36 \%$ & $92 \%$ \\
Urbano-Privado & $90 \%$ & $28 \%$ & $97 \%$ \\
Rural & $86 \%$ & $31 \%$ & $94 \%$ \\
\hline Total & $89 \%$ & $31 \%$ & $93 \%$ \\
\hline
\end{tabular}

Fuente: Cálculos propios de datos procedentes de la encuesta administrada por el Ministerio de Educación y la Oficina Regional de UNESCO para América Latina y el Caribe (Laboratorio Latinoamericano de la Calidad de la Educación). Estrato Mega Ciudad se refiere a ciudades con más de 1 millón de habitantes. Urbano se refiere a ciudades de menos de 1 millón de habitantes.

Al preguntarles qué es lo que menos les gusta de la escuela, uno de cada cinco estudiantes indicaron

que los compañeros, y una proporción semejante señaló que los maestros.

Cuadro 16. Respuesta de los estudiantes a la pregunta de qué es lo que más les molesta de la escuela.

\begin{tabular}{|lcccccc|}
\hline & Compañeros & Maestros & Director & Aula & Patio & Otros \\
\hline Mega-Público & $24 \%$ & $24 \%$ & $8 \%$ & $11 \%$ & $25 \%$ & $9 \%$ \\
Mega-Privado & $18 \%$ & $24 \%$ & $9 \%$ & $14 \%$ & $25 \%$ & $10 \%$ \\
Urbano-Público & $33 \%$ & $19 \%$ & $9 \%$ & $17 \%$ & $16 \%$ & $6 \%$ \\
Urbano-Privado & $20 \%$ & $26 \%$ & $11 \%$ & $15 \%$ & $18 \%$ & $11 \%$ \\
Rural & $26 \%$ & $15 \%$ & $8 \%$ & $20 \%$ & $25 \%$ & $6 \%$ \\
\hline Total & $26 \%$ & $20 \%$ & $9 \%$ & $17 \%$ & $21 \%$ & $7 \%$ \\
\hline
\end{tabular}

Fuente: Cálculos propios de datos procedentes de la encuesta administrada por el Ministerio de Educación y la Oficina Regional de UNESCO para América Latina y el Caribe (Laboratorio Latinoamericano de la Calidad de la Educación). Estrato Mega Ciudad se refiere a ciudades con más de 1 millón de habitantes. Urbano se refiere a ciudades de menos de 1 millón de habitantes.

Quienes pelean constantemente con sus compañeros tienen una probabilidad $50 \%$ superior de mencionar que lo que menos les gusta de la escuela son sus compañeros que quienes no tienen peleas constantes. Mientras que entre quienes pelean constantemente, el $34 \%$ dice que sus compañeros son lo que menos les gusta de la escuela, entre quienes no pelean constantemente, el $22 \%$ dice que lo que menos les gusta son sus compañeros.

Igualmente, los alumnos que dicen no confiar en sus maestros tienen una probabilidad $40 \%$ más alta de señalar que lo que menos les gusta de la escuela son los maestros que aquellos que sí confían en sus maestros. Mientras que entre los que dicen que sí confían en sus maestros el $16 \%$ señala que los maestros es lo que menos les gusta de la escuela, entre quienes dicen que a veces confían en sus maestros el $20 \%$ señalan a los maestros como lo que menos les gusta de la escuela, y entre quienes dicen que no confían en sus maestros el $23 \%$ señalan a los maestros como lo que menos les gusta de la escuela. En cualquiera de los casos es sintomático que uno de cada cinco estudiantes señale que son sus maestros lo que menos les gusta de su escuela.

Al preguntar a los padres cuáles son los principales problemas de la escuela una tercera parte señalan robos, uno de cada cinco se refieren a violencia entre alumnos y una tercera parte se refieren a falta de disciplina. Todos estos problemas coinciden en caracterizar un clima social y afectivo deficiente, de falta de respeto por los demás, de poca capacidad de resolver diferencias en forma productiva y pacífica.

Estas deficiencias en el clima social que la escuela brinda a los alumnos se relacionan con el clima social que caracteriza las relaciones entre maestras y directores. Una proporción importante de las escuelas no funcionan como organizaciones colegiadas integradas. Así, uno de cada cuatro maestros no considera que sus colegas apoyan sus iniciativas, uno de cada cinco no siente que el director de la escuela 
reconoce su trabajo y una tercera parte indica que no hay comunicación entre colegas sobre tareas pedagógicas. Uno de cada cuatro con-

sidera que no son claros los roles entre el director y los maestros y un $17 \%$ se siente inseguro en la escuela.

Cuadro 17. Respuestas de los padres a la pregunta de cuáles son los problemas más graves de la escuela.

\begin{tabular}{|lcccccr|}
\hline & $\begin{array}{c}\text { Mega- } \\
\text { público }\end{array}$ & $\begin{array}{c}\text { Mega- } \\
\text { privado }\end{array}$ & $\begin{array}{r}\text { Urbano- } \\
\text { público }\end{array}$ & $\begin{array}{l}\text { Urbano- } \\
\text { privado }\end{array}$ & Rural & Total \\
\hline $\begin{array}{l}\text { Robos } \\
\text { Violencia entre }\end{array}$ & $44.2 \%$ & $26.5 \%$ & $34.9 \%$ & $32.1 \%$ & $25.4 \%$ & $32.0 \%$ \\
$\begin{array}{l}\text { alumnos } \\
\text { Consumo de }\end{array}$ & $24.6 \%$ & $21.7 \%$ & $22.2 \%$ & $21.2 \%$ & $17.1 \%$ & $20.7 \%$ \\
$\begin{array}{l}\text { drogas } \\
\begin{array}{l}\text { Falta de disciplina } \\
\text { Consumo de alcohol }\end{array}\end{array}$ & $1.0 \%$ & $.9 \%$ & $1.6 \%$ & $1.3 \%$ & $1.6 \%$ & $1.4 \%$ \\
$\begin{array}{l}\text { Alumnos sin interés } \\
\text { en estudios }\end{array}$ & $.3 \%$ & $36.1 \%$ & $27.9 \%$ & $21.8 \%$ & $39.9 \%$ & $31.4 \%$ \\
$\begin{array}{l}\text { Profesores abusan } \\
\text { de alumnos }\end{array}$ & $4.7 \%$ & $7.4 \%$ & $9.9 \%$ & $14.7 \%$ & $11.1 \%$ & $9.7 \%$ \\
$\begin{array}{l}\text { Profesores } \\
\text { desmotivados }\end{array}$ & $.3 \%$ & $.9 \%$ & $1.1 \%$ & $1.3 \%$ & $.5 \%$ & $.8 \%$ \\
$\begin{array}{l}\text { Poca consideración } \\
\text { con padres }\end{array}$ & $1.3 \%$ & $3.0 \%$ & $1.3 \%$ & $1.3 \%$ & $3.1 \%$ & $2.0 \%$ \\
\hline
\end{tabular}

Fuente: Cálculos propios de datos procedentes de la encuesta administrada por el Ministerio de Educación y la Oficina Regional de UNESCO para América Latina y el Caribe (Laboratorio Latinoamericano de la Calidad de la Educación). Estrato Mega Ciudad se refiere a ciudades con más de 1 millón de habitantes. Urbano se refiere a ciudades de menos de 1 millón de habitantes.

Cuadro 18. Caracterización que hacen los maestros del clima organizacional en la escuela.

\begin{tabular}{|c|c|c|c|c|c|}
\hline & $\begin{array}{c}\text { Mis colegas } \\
\text { apoyan mis } \\
\text { iniciativas }\end{array}$ & $\begin{array}{l}\text { El director } \\
\text { reconoce } \\
\text { mi trabajo }\end{array}$ & $\begin{array}{c}\text { Mis colegas } \\
\text { me consultan } \\
\text { sobre el } \\
\text { trabajo }\end{array}$ & $\begin{array}{c}\text { Falta } \\
\text { definición } \\
\text { de roles } \\
\text { dirección } \\
\text { y maestros }\end{array}$ & $\begin{array}{c}\text { La } \\
\text { inseguridad } \\
\text { en la escuela } \\
\text { me pone } \\
\text { nervioso }\end{array}$ \\
\hline Mega-Público & $68.20 \%$ & $76.50 \%$ & $62.20 \%$ & $21.50 \%$ & $22.30 \%$ \\
\hline Mega-Privado & $77.50 \%$ & $83.00 \%$ & $73.50 \%$ & $22.90 \%$ & $8.40 \%$ \\
\hline Urbano-Público & $74.00 \%$ & $81.90 \%$ & $65.90 \%$ & $31.20 \%$ & $22.30 \%$ \\
\hline Urbano-Privado & $73.90 \%$ & $82.10 \%$ & $66.60 \%$ & $20.50 \%$ & $6.60 \%$ \\
\hline Rural & $78.10 \%$ & $80.80 \%$ & $65.00 \%$ & $33.50 \%$ & $13.50 \%$ \\
\hline Total & $74.50 \%$ & $80.80 \%$ & $65.80 \%$ & $27.90 \%$ & $16.90 \%$ \\
\hline
\end{tabular}

Fuente:Cálculos propios de datos procedentes de la encuesta administrada por el Ministerio de Educación y la Oficina Regional de UNESCO para América Latina y el Caribe (Laboratorio Latinoamericano de la Calidad de la Educación). Estrato Mega ciudad se refiere a ciudades con más de 1 millón de habitantes. Urbano se refiere a ciudades de menos de 1 millón de habitantes.

En síntesis, el clima en una proporción importante de escuelas en Colombia es, a los ojos de estudiantes y maestros, deficiente desde el punto de vista de la convivencia. No hay respeto y hay demasiada violencia y malas relaciones interpersonales entre alumnos. Los maestros y directores, por su parte, tampoco tienen entre sí relaciones interper- sonales y colegiadas respetuosas para permitir la que el trabajo en equipo sea productivo. Como consecuencia, es improbable que en escuelas con estas deficiencias en el clima social puedan desarrollarse habilidades y disposiciones fundamentales para vivir en democracia: la capacidad de respetar a otras personas, la posibilidad de valorar las diferencias como una fortaleza, la habilidad para negociar diferencias de forma pacífica y productiva. Al mismo tiempo, el deficiente clima social de muchas escuelas condena a los profesores a tratar de enfrentar solos los complejos desafíos de enseñar a niveles de excelencia a estudiantes, en medio de carencias de condiciones materiales básicas y de limitaciones en sus propias capacidades para hacerlo.

\section{Propuestas para la acción: fortalecer las competencias pedagógicas de los maestros y desarrollar fuertes culturas escolares donde el respeto a los demás y el aprecio por la libertad sean valores que se reflejen en las relaciones cotidianas}

No hay solución simple a los complejos desafíos de deficiencias pedagógicas y organizacionales descritas en este trabajo para apoyar el que los estudiantes en Colombia aprendan en la escuela en profundidad habilidades y disposiciones que les permitan ejercer ser ciudadanos responsables y vivir en paz. ¿Cómo pueden aprender a confiar en los demás estudiantes que no sienten que pueden confiar en sus maestros, en sus compañeros y que describen que sus escuelas son ambientes violentos? ¿Cómo hacer que no deserten de la escuela estudiantes que encuentran poco claras las explicaciones de sus profesores, para quienes lo más desagradable de la escuela son compañeros y profesores? ¿Cómo hacer que aprendan a leer a niveles sofisticados estudiantes cuyos profesores no leen de forma estimulante, con insuficientes materiales de lectura, en cuyos hogares no hay costumbre de leer ni materiales de lectura?

El intentar resolver estos problemas requiere el esfuerzo conjunto y coordinado de diversos grupos, y la colaboración efectiva a nivel local y en niveles superiores de administración. En la escuela es importante 
que las escuelas comiencen a funcionar como equipos de trabajo, donde maestros y directores colaboren, se comuniquen efectivamente, se respeten, y modelen para sus estudiantes un clima democrático y de gestión grupal eficaz. Para que los maestros puedan hacer esto probablemente necesiten apoyo de otras instancias, apoyo e incentivos para desarrollar competencias pedagógicas y de trabajo en grupo, materiales pedagógicos para poder facilitar su tarea, y esfuerzos y una relación con niveles superiores de administración que comuniquen claras expectativas y respeto hacia la profesión docente. Sólo los maestros que experimenten un trato respetuoso de sus colegas y superiores podrán a su vez aprender a respetar a sus colegas y estudiantes, sólo quienes experimenten relaciones democráticas podrán construir climas democráticos en sus escuelas, sólo quienes comprendan que sobre ellos hay altas expectativas y esperanza podrán a su vez tener sobre sus estudiantes altas expectativas y esperanzas.

El cambio de aquellos aspectos más importantes en el proceso educativo, es decir, de las prácticas que constituyen la cotidianeidad de la escuela, tiene sólo vínculos muy tenues con las decisiones de los planificadores educativos, y depende mucho más de las decisiones, expectativas y de la práctica de múltiples agentes en la escuela y alrededor de ella que son independientes de los planificadores y pueden ser igualmente transformadas de forma significativa en el curso de su implantación.

La práctica educativa tiene mucho más que ver con la cultura de la escuela, con la forma en que maestros definen su rol, con las expectativas recíprocas entre maestros, directivos escolares, en ocasiones miembros de la comunidad, que con la práctica de los planificadores educativos tradicionales. Los cambios en educación ocurren como resultado de la construcción compartida

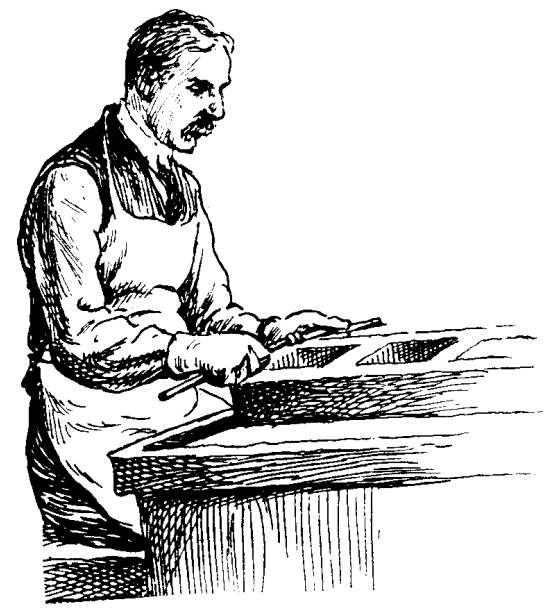

entre un grupo grande de interlocutores, por lo que es necesario construir espacios explícitamente de articulación de este diálogo para poder examinar las consecuencias de las prácticas actuales y considerar posibles prácticas alternativas ${ }^{11}$.

La mejora de las condiciones de vida en América Latina descansa en parte en la profundización de la democracia, en la construcción de comunidad, del tejido social, en el fortalecimiento de las capacidades asociativas y de la confianza interpersonal, así como en el aumento de las competencias individuales que hagan a las personas más eficaces y productivas. Las políticas educativas consistentes con ese propósito tienen que ser necesariamente incluyentes, no pueden continuar manteniendo sistemas de apartheid educativo donde muchos aprenden muy poco. Además, se debe plantear una visión amplia del tipo de competencias necesarias para construir comunidades y sociedades democráticas. Hay competencias individuales necesarias para esto como el desarrollar habilidades interpersonales en resolución de conflictos o el desarrollo del pensamiento moral. Hay experiencias

\footnotetext{
${ }^{11}$ Ver Reimers, Fernando y McGinn, Noel, 2000. Diálogo informado. México: Centro de Estudios Educativos y Asociación de Universidades Encomendadas a la Compañía de Jesús en América Latina.
}

que tienen que ser integradas en el currículo si la escuela ha de servir a formar ciudadanos que puedan ejercer prácticas democráticas en su vida cotidiana. Un proyecto incluyente, orientado a la equidad tiene más probabilidades de mejorar las condiciones de vida de todas las personas.

Quienes entienden que una función importante de la escuela es la de brindar igualdad de oportunidades educativas a todos los niños, reconocen que esto significa más que brindar igual oportunidad de matricularse en primer grado ${ }^{12}$. Es esencial apoyar con educación de calidad a los estudiantes a lo largo de todas sus trayectorias educativas ${ }^{13}$. Para revitalizar la cultura de la escuela de forma que las maestras se sientan apoyadas por sus colegas, y para que todas trabajen en búsqueda de un propósito común, es necesario iniciar trabajo en equipo que se apoye en métodos de investigación acción, donde equipos de maestras se reúnan a discutir los desafíos que confrontan sus estudiantes, a identificar sus causas y a establecer programas escolares de cambio y programas de autoformación. En la última década se ha experimentado en América Latina con múltiples programas piloto orientados a que los equipos docentes lleven a cabo formas diversas de diagnóstico y planeación de este tipo. El desafió en muchos casos es orientar estos esfuerzos de gestión escolar hacia el desarrollo de pedagogías más efectivas.

Es necesario entender el desarrollo profesional de los maestros como un proceso de largo plazo que re-

\footnotetext{
12 Esta noción de que la igualdad de oportunidades equivale a la igualdad en la oportunidad de acceso inicial a la escuela domina el ideario público sobre educación en la región. 13 Para una discusión detallada de las políticas orientadas a fortalecer la equidad, véase Reimers, F. 2001. Editor. Unequal Schools, Unequal Chances. The challenges to equal opportunity in the Americas. Cambridge, MA. Harvard University Press. Publicado en español como Distintas Escuelas. Diferentes oportunidades. Madrid: Editorial Arco/La Muralla.
} 
sulta de la preparación académica, de las experiencias acumuladas a través de los años, y de examinar sistemáticamente sus prácticas en el aula. Este proceso de largo plazo incluye oportunidades y experiencias que se planifican sistemáticamente.

El desarrollo profesional de los docentes puede considerarse desde una perspectiva tradicional o contemporánea. La perspectiva contemporánea concibe al desarrollo profesional basado en una perspectiva constructivista, como proceso de largo plazo, donde los maestros son percibidos como estudiantes activos, y donde el apoyo y seguimiento sistemáticos son indispensables. El desarrollo profesional está integrado con actividades diarias y relacionado con esfuerzos más amplios de reforma escolar. Esta perspectiva entiende a los maestros como investigadores con capacidad de reflexionar sobre sus prácticas. La perspectiva constructivista, entiende al conocimiento como continuamente en formación y como el resultado del trabajo conjunto entre docentes y aprendices. En esta perspectiva el desarrollo profesional es un proceso de colaboración en equipos y existe una gran variedad de modelos para promoverlo. El contexto determina cuál es la "combinación” más efectiva.

En comparación, la perspectiva tradicional está basada en un modelo de transmisión del conocimiento, las oportunidades de formación son de corto plazo y están desconectadas; en esta perspectiva los maestros son pasivos e interpretan el conocimiento generado por otros y generalmente hay poco apoyo o seguimiento. En esta perspectiva, el desarrollo profesional está desconectado de la práctica cotidiana, no se concibe como parte de la reforma educativa. Esta perspectiva entiende a los maestros como consumidores e interpretes de las investigaciones y teorías de otros

Características del desarrollo profesional de los maestro. En esta perspectiva el desarrollo profesional puede ser un proceso individual o de colaboración. El contexto raramente se toma en cuenta para definir cuál es la modalidad más adecuada de formación.

Cuáles son las competencias que necesitan desarrollar los maestros?

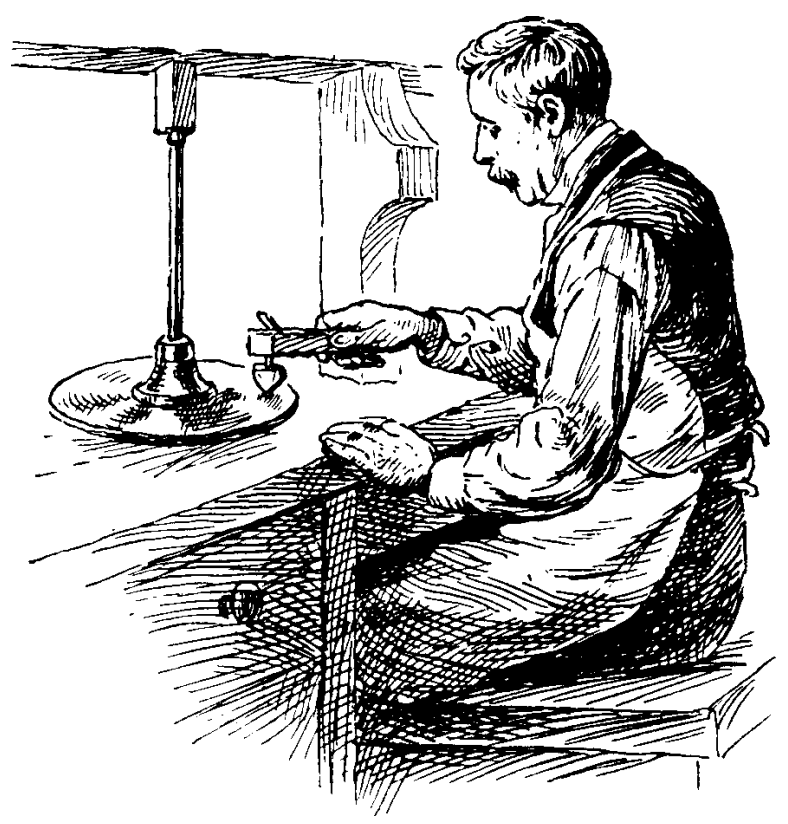

- Materias a enseñar.

- Conocimiento de pedagogía como materia.

- Conocimiento de la práctica pedagógica.

- Conocimiento del contexto de los estudiantes.

- Psicología del desarrollo humano.

- Destrezas para relacionar la teoría con la práctica.

- Destrezas clínicas (preguntar, escuchar, etc.).

- Destrezas para usar y enseñar sobre la tecnología.

- Destrezas de evaluación y asesoría.

- Conocimiento de la escuela, su comunidad y su sociedad.

- Conciencia política y de justicia social.

Hay varios modelos para el desarrollo profesional de los maestros, los cuales pueden agruparse en dos grandes categorías: aquellos que requieren colaboración entre instituciones, y aquellos que se basan en la acción en grupos pequeños, aquellos que requieren.

\section{Modelos que requieren colaboración entre instituciones}

- Escuelas de desarrollo profesional.

- Otras colaboraciones entre escuelas y universidades.

- Colaboración entre universidades, escuelas y otras instituciones.

- Redes de escuelas.

- Redes de maestros.

Educación a distancia

\section{Modelos individuales o de pequeños grupos}

- Supervisión: tradicional y clínica

- Asesoría de las actividades de los estudiantes.

Seminarios, cursos, talleres, etc.

- Estudios de caso.

- Desarrollo dirigido individualmente. 
- Desarrollo con colegas.

- Observación de prácticas excelentes.

- Participación en roles nuevos.

- Uso de narrativas.

- Uso de tutores o mentores.

- Modelo generacional.

- Modelo de desarrollo de destrezas.

- Modelos de reflexión

- Modelos basados en proyectos

- Portafolios.

- Investigación acción.

Es importante que en estas tareas de apoyo a la formación de equipos de docentes en la escuela, el trabajo de los docentes se centre tanto en las cuestiones de técnica pedagógica, de cómo enseñar mejor y en profundidad las habilidades fundamentales como la lectoescritura, como en conversaciones más profundas sobre los valores que debe reflejar la escuela y sobre cómo preparar a los estudiantes para vivir en democracia. Es conveniente que los maestros discutan en qué forma la organización de la escuela y las formas de relación entre profesores y estudiantes son en sí mismas poderosas lecciones morales. Es difícil enseñar a vivir en democracia si las relaciones en la escuela son autoritarias e irrespetuosas. El desarrollo de competencias para el respeto a la legalidad, a la vida de los demás, a las instituciones, requiere atención a tres áreas ínter-relacionadas: la construcción de ambientes democráticos en la escuela, la inclusión en el currículo de atención a los procesos para el desarrollo de competencias para la convivencia y, finalmente, la atención a la relación de los equipos docentes con comunidades y con administradores y supervisores. Es insuficiente tratar de desarrollar competencias para la convivencia en una asignatura específica, si éstas contradicen la cultura más amplia de la escuela. No tendrán efecto espacios aislados en el programa de estudio para desarrollar la sensibilidad ciudadana si estos están inmersos en culturas escolares autoritarias e irrespetuosas que contradigan en su práctica los mensajes de estas asignaturas. Es necesario asegurar que las disposiciones y destrezas para la vida en democracia se desarrollen simultáneamente en el currículo más explícito -en las asignaturas-, en el currículo más profundo de la escuela -reflejado en la forma en que se relacionan alumnos entre sí, alumnos con profesores- y en la forma en que los equipos colegiados a su vez se relacionan con los padres y la comunidad escolar y con los supervisores escolares y otras instituciones de la localidad.

Los grupos de profesores en las escuelas que conversen sobre la forma de mejorar su práctica podrían discutir cómo preparar a los estudiantes para la vida, ayudarles a desarrollar destrezas que permitan desarrollar la capacidad de pensar por cuenta propia, desarrollar el aprecio por la libertad y la capacidad para ejercerla con responsabilidad, desarrollar la capacidad de apreciar la diversidad y las diferencias entre las personas, desarrollar la capacidad de asociarse con los demás, desarrollar la capacidad de valorar los derechos fundamentales de todas las personas, desarrollar la capacidad de vivir saludablemente y de preservar el equilibrio ecológico y de-

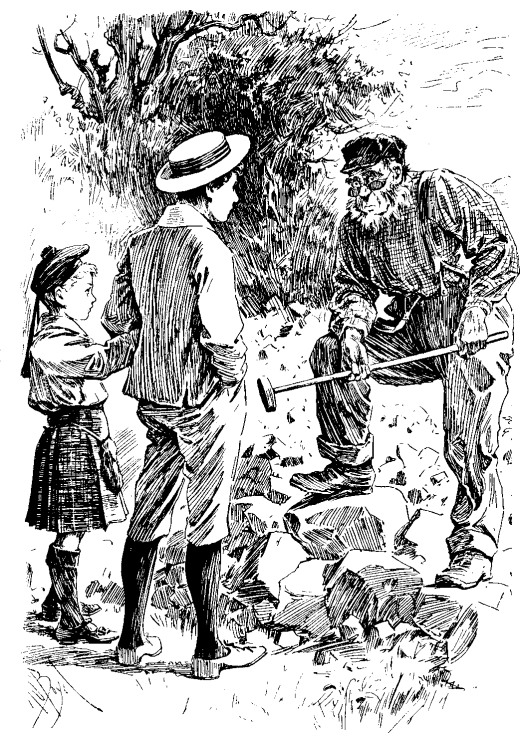

sarrollar capacidades sofisticadas de comunicación y de aprender continuamente. El desarrollo de estas capacidades es esencial a un proyecto de sociedad orientado a la libertad, a la democracia y a la justicia. En medio de una de las recesiones económicas más severas de las últimas décadas, es conveniente que la discusión sobre los propósitos de la educación no se reduzca demasiado al desarrollo de competencias con rentabilidad en el mercado laboral. Es conveniente insistir en que el aprender es un bien en sí mismo y que reduce una forma de pobreza, aun si no se traduce en mejoras materiales en las condiciones de vida de las personas.

El currículo de las escuelas debe desarrollar competencias específicas para la vida en democracia. Como resultado de la pesada herencia autoritaria la mayoría de las escuelas de la región son mudas en relación a qué hace falta para vivir en democracia. La mayoría ni siquiera enseña a los estudiantes cuáles son los derechos humanos contenidos en la declaración universal adoptada hace más de medio siglo por las naciones del mundo en la creación de Naciones Unidas. Como resultado, las escuelas producen analfabetos cívicos. Entre las competencias más importantes que las escuelas deberán desarrollar, están las de poder negociar diferencias con otros, las de saber apreciar la diversidad como fortaleza, las de tener repertorios efectivos de comunicación interpersonal y grupal y de logros de acuerdos. Los programas educativos deben explícitamente incorporar contenidos que desarrollen habilidades, disposiciones y valores que prefieran la resolución pacífica de conflictos a las alternativas, que reconozcan y valoren la diversidad de puntos de vista, que prefieran las formas democráticas de organización a las formas autoritarias, que permitan construir una cultura cívica desde la escuela donde el recurso a la argumentación razonada sobre la base de evidencia sea preferido como for- 
ma de lograr acuerdos y de persuadir a la imposición de dogmas de fe por unos grupos sobre otros.

Es factible apoyar a las maestras en la tarea de enseñar a los niños a leer y de desarrollar competencias cívicas. En pequeña escala existen experimentos en la región y fuera de ella que permitirían a cada maestra conocer cómo desarrollar una clase efectiva en cada uno de estos temas. Hay programas que han demostrado ser efectivos desarrollando habilidades de lecto-escritura, curricula sobre derechos humanos, programas que promueven la lectura crítica de la prensa en comunidades marginadas. Una primera tarea aún por hacer es hacer un inventario de estos programas, catalogarlos y hacer este catálogo accesible a quien tenga interés. Una segunda es promover el desarrollo de materiales impresos de alta calidad que permitan apoyar a las maestras al implementar estos programas en el aula.

Hay en Colombia una rica experiencia con buenos materiales para la promoción de la vida democrática, las actitudes cívicas, la discusión de dilemas éticos, desarrollados por Fundaciones, Universidades y por algunas Secretarias de Educación, como la Secretaría de Educación de Bogotá, en años recientes. Algunos de estos materiales incluyen los publicados en la serie de revistas de la Fundación Restrepo Barco sobre liderazgo, participación, enfoque de

\footnotetext{
${ }^{14}$ Entre otras: "El liderazgo y la comunicación efectiva: punto de partida para una óptima gestión administrativa", por Gloria Cajiao de Pérez y Yolanda Sierra Castellanos. F.R.B., UNICEF Colombia, I.C.B.F. Bogotá. 2001; "La participación está en juego", por María Victoria Estrada, Edda Madrid-Malo Luz Marina Gil. F.R.B., UNICEF Colombia. Bogotá. 2000; "Los derechos de la niñez. Una visión integral en procesos de atención", por Nelson Ortiz Pinilla. F.R.B., UNICEF Colombia. 1997; "La dimensión ético de los proyectos sociales el sentido-, por Esmeralda Ruiz González. ICBF, F.R.B., Fundación FES UNICEF Colombia. Bogotá. 2001; "Familia y Comunidad". En el marco de la protección integral por Catalina Turbay Restrepo. F.R.B. UNICEF Colombia. Diciembre, Bogotá, 1997;
}

género, y desarrollo organizacional ${ }^{14}$. La compañía de Jesús ha desarrollado un conjunto de cartillas para el desarrollo de habilidades para la resolución pacífica de conflictos ${ }^{15}$; también el Instituto Luis Carlos Galán ha desarrollado cartillas con fines semejantes ${ }^{16}$. La Secretaría de Educación de Bogotá desarrolló pruebas de comprensión y sensibilidad ciudadana en 1998 para evaluar los conocimientos y la sensibilidad de los estudiantes que son útiles para conocer variación entre escuelas, y para promover discusión entre equipos docentes en una escuela en relación a cómo desarrollar estas disposiciones y conocimientos. Las Escuelas de Perdón y Reconciliación en varios barrios de Bogota también han adquirido experiencia valiosa en el desarrollo de metodologías para promover la noviolencia.

Ejemplos de sitios en la red donde hay catálogos de materiales para enseñanza de habilidades para la vida en democracia y para la resolución pacifica de conflictos desarrollados en otras latitudes son:

"Niña azul niño rosa. Reflexiones sobre e enfoque de género en el proceso de formación de niñas y niños", por Mónica Tobón Coral. F.R.B., UNICEF Colombia, I.C.B.F., Fundación FES. Abril. Bogotá. 2001; "Régimen jurídico para instituciones de protección", por Faridy Jiménez Valencia y Gloria Elena Ochoa. Instituto SER de Investigación, F.R.B. Mayo. Bogotá. 1997; "Elementos conceptuales básicos. Manual para facilitadores y formadoras", por Gloria Elena Ochoa y Maribel Delgadillo. Instituto SER de Investigación, F.R.B. Junio, Bogotá, 1997; "La información. Estrategia para el crecimiento de la organización", por María Victoria Estrada y Carmenza González de Arenas. Fundación Rafael Pombo, F.R.B. Junio, Bogotá, 1997; "Una mirada a las niñas en las instituciones de protección", por Paulina Ospina Mallarino. UNICEF Colombia, F.R.B. Diciembre, Bogotá, 1997. Para mas información contactar vease www.funrestre pobarco.org.co

${ }^{15}$ Programa por la paz. Compañía de Jesús. Proceso de formación. Desarrollo de habilidades para la construcción de la paz. Cartillas 1,2 , y 3 . propazjunete.com

16 "Resolución democrática de los conflictos en la escuela", por Rodrigo Uprimny, Guido A. Bonilla, Juan G. Gómez. Instituto para el desarrollo de la democracia Luis Carlos Galán, UNICEF Colombia. Febrero, Bogotá, 1998. sdc@ilcg.colnodo. apc.org

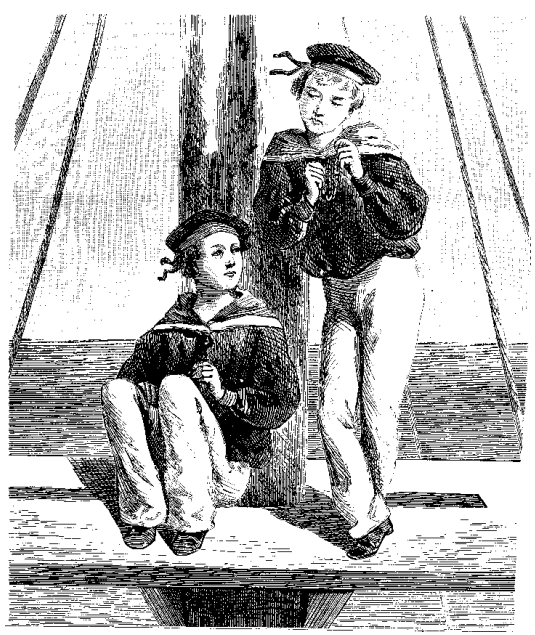

Civitas International www.civnet.org International Bureau of Education www.ibe.unesco.org/regional/ diversity

Center for Social and Emotional

Learning. www.csee.net

Carácter Education Partnership.

www.character.org

Creative Responses to Conflict

www.ccrcglobal.org

Facing History and Ourselves

www.facinghistory.org

Human Rights Education

Association www.hrea.org

Fund for Global Awakening

www.ffga.org

Peace Games

www.peacegames.org

Resolving Conflict Creatively

www.esrnational.org

Seeds of Peace

www.seedsofpeace.org

Voices of Love and Freedom

www.naschools.org

Workable Peace

www.workablepeace.org

La tarea de desarrollar competencias cívicas en la escuela debe ser apoyada por acciones complementarias fuera de la escuela. Esto significa varias cosas interrelacionadas. En primer lugar, que el diálogo sobre los propósitos a los que ha de servir la educación, ha de ser un diálogo abierto a la participación de la ciudadanía. Las políticas educativas han de ser concebidas reflejando la 
pluralidad de intereses legítimos en una sociedad moderna. En particular, es necesario identificar y desarrollar mecanismos para que los más pobres puedan participar en expresar sus preferencias en relación a que propósitos para la educación de sus hijos. Sería estupendo que los grupos locales de investigación que están apoyando el diálogo de la Alianza entrevistaran a grupos de padres de familia, a desertores escolares y a estudiantes para conocer qué esperan ellos de la escuela, que destrezas y habilidades quieren aprender, en qué medida lo que se aprende en la escuela es pertinente a las necesidades que ellos tienen. El diseño y la implementación de la política educativas han de ser oportunidades cotidianas para desarrollar competencias de negociación, de reconocimiento de intereses diversos, de identificación de oportunidades de mutuo beneficio, de aprender a confiar en los demás a través de experiencias compartidas de resolver pacífica y productivamente diferencias en el desarrollo de proyectos concretos para actualizar el potencial de todas las personas.

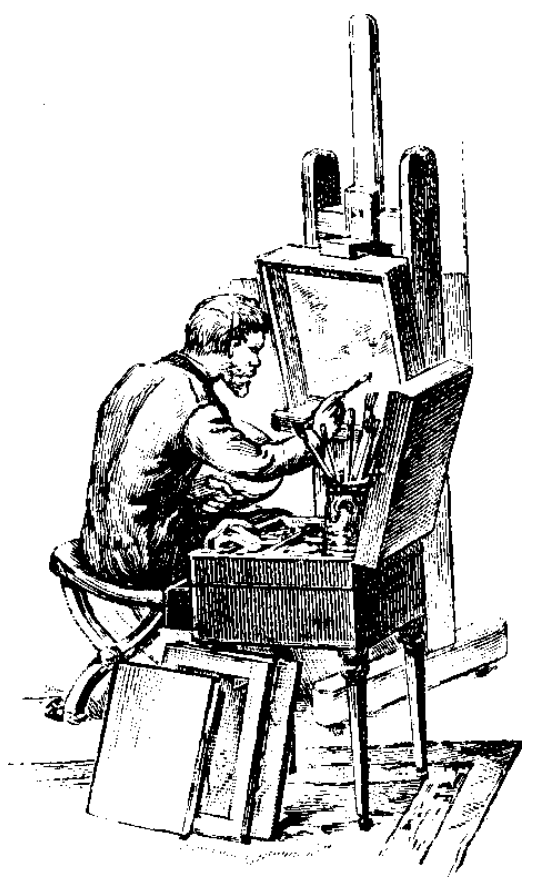

En segundo lugar, es necesario que la administración de los sistemas educativos esté abierta al escrutinio público. Esto implica también el desarrollo de formas de colaboración con organizaciones de la sociedad civil, con organizaciones no gubernamentales, asociadas con las escuelas públicas en el mejoramiento de la oferta educativa. También desarrollar formas de participación de la comunidad en la gestión educativa, más como forma de desarrollar capital social a nivel local que como estrategia para mejorar la efectividad pedagógica de los centros.

El carácter centralista de muchas decisiones de política educativa, pesado legado de la larga herencia autoritaria de América Latina, y la ausencia de una cultura de evaluación pública de los resultados de las políticas educativas, cercenan la posibilidad de construir una agenda pública -la cual debería ser el resultado de un modelo pluralista de negociación entre intereses en competencia- en la que estén representados los intereses de los beneficiarios de la educación y en particular los de los más pobres.

Pero para que estas ideas sean verdaderamente públicas, para que no sean dominio particular de grupos limitados de funcionarios educativos, es esencial que las mismas se inserten en procesos de discusión pública, de diálogo democrático, que contribuyan a hacer al público cada vez más sofisticado en su conocimiento y comprensión de los problemas educativos, así como de las opciones para intervenir sobre estos problemas.

En síntesis, los desafíos para que los estudiantes en zonas rurales aprendan en profundidad contenidos y disposiciones que aumenten sus opciones en la vida, dependen de que sus maestras puedan enseñarles mejor. Para ello, serán necesarios esfuerzos deliberados y de gran envergadura para desarrollar las competencias de las maestras, atención a la provisión de materiales de aprendizaje, libros de texto, guías de aprendizaje y otros recursos didácticos, y la renovación de culturas escolares donde los profesores puedan trabajar en grupo para identificar soluciones locales que permitan a sus estudiantes aprender, tal y como hicieron en el principio de esta maravillosa innovación que hoy celebramos, Oscar Mogollón y un grupo de maestros que trabajaban en escuelas rurales en Colombia. En el camino será útil pensar con amplitud sobre el tipo de disposiciones que prepararan a los estudiantes para vivir en paz y ser libres. El éxito en el proceso dependerá de una combinación efectiva de innovación desde la escuela y de presión y apoyo desde los gobiernos y la sociedad civil, acompañada de muchos amigos críticos y de oportunidades como esta, para hablar claro y sin tapujos.

\section{Bibliografía}

Beaton, A., I. Mullis, M. Martin, E. González, D. Kelly and T. Smith. Mathematics Achievement in the Middle school Years: IEA's Third International Mathematics and Science Study (TIMSS). International Association for the Evaluation of Educational Achievement. Chestnut Hill, MA. Boston College, 1996.

Science Achievement in the Middle school Years: IEA's Third International Mathematics and Science Study (TIMSS). International Association for the Evaluation of Educational Achievement. Chestnut Hill, MA. Boston College, 1996.

Reimers, Fernando. "La lucha por la igualdad de oportunidades educativas en América Latina como proceso político". En Revista Latinoamericana de Estudios Educativos. Vol. 32. № 1, 2002, págs. 9-70.

ReIMERS, F., McGinn. Diálogo informado. México, Centro de Estudios Educativos y Asociación de Universidades Encomendadas a la Compañía de Jesús en Amèrica, 2000.

Sarmiento Gómez, Alfredo. "Política y equidad educativas en Colombia". En Reimers, F. Distintas escuelas, diferentes oportunidades. Los retos para la igualdad de oportunidades en Latinoamérica. Madrid, Editorial Arco, 2002.

Sarmiento Gómez, A.; L. Tovar y C. Alam. Situación de la Educación Básica, Media y Superior. Casa Editoral EI Tiempo, Bogotá, 2002. 